\title{
Microwave-Promoted Pd-Catalyzed Cyanation of Aryl Triflates: A Fast and Versatile Access to 3- Cyano-3-desoxy-10-ketomorphinans
}

\author{
Ao Zhang and John L. Neumeyer* \\ Alcohol and Drug Abuse Research Center, McLean Hospital, Harvard Medical School, \\ 115 Mill Street, Belmont, MA 02478
}

Neumeyer@mclean.harvard.edu

\section{Experimental Section}

General: ${ }^{1} \mathrm{H}$ and ${ }^{13} \mathrm{C}$ NMR spectra were recorded at 300 and $75 \mathrm{MHz}$, respectively, on a Bruker AC300 spectrometer. Chemical shifts are given as $\delta$ value (ppm) downfield from tetramethylsilane as an internal reference. Melting points were determined on a Thomas-Hoover capillary tube apparatus and are reported uncorrected. Element analyses, performed by Atlantic Microlabs, Atlanta, GA, were within $\pm 0.4 \%$ of theoretical values. Analytical thin-layer chromatography (TLC) was carried out on 0.2-mm Kieselgel 60F 254 silica gel plastic sheets (EM Science, Newark). Flash chromatography was used for the routine purification of reaction products. The column output was monitored with TLC.

Microwave heating was performed in a SmithSynthesizer ${ }^{\mathrm{TM}}$ single mode cavity, producing continuous irradiation at $2450 \mathrm{MHz}$. Reaction temperature and pressure were determined using the built-in, on-line IRand pressure sensors.

\section{General Procedure ${ }^{1}$ for Synthesis of Aryl triflates:}

3-Hydroxy-N-alkyl-10-ketomorphinan ${ }^{2} \quad(1.53 \mathrm{mmol})$, which was prepared from levorphanol, was dissolved in anhydrous $\mathrm{CH}_{2} \mathrm{Cl}_{2}(10 \mathrm{~mL})$ and $\mathrm{Et}_{3} \mathrm{~N}(4 \mathrm{~mL})$. The mixture was cooled to $0^{\circ} \mathrm{C}$, and then $\mathrm{PhNTf}_{2}(1.0 \mathrm{~g}, 2.80 \mathrm{mmol})$ was added. The reaction mixture was allowed to warm to

(1) Wentland, M. P.; Xu, G.; Cioffi, C. L.; Ye, Y.; Duan, W.; Cohen, D. J.; Colasurdo, A. M.; Bidlack, J. M. Bioorg.\& Med. Chem. Lett. 2000, 10, 183-187.

(2) Neumeyer, J. L.; Bidlack, J. M.; Zong, R. S.; Bakthavachalam, V.; Gao, P.; Cohen, D. J.; Negus, S. S.; Mello, N. K. J. Med. Chem. 2000, 43, 114-122. room temperature overnight. The solution was diluted with $\mathrm{CH}_{2} \mathrm{Cl}_{2}$, washed with $1 N \mathrm{NaOH}$ followed by brine, and then dried over anhydrous sodium sulfate. The solvent was removed in vacuo to afford a crude product, which was purified by column chromatography on silica gel (Hexane:ethyl acetate) to give the corresponding triflate.

\section{(-)-3-Trifluoromethanesulfonyloxy-10-keto-N-}

cyclobutylmethylmorphinan (1a) was obtained as a semisolid in $85.8 \%$ yield. ${ }^{1} \mathrm{H} \mathrm{NMR}\left(\mathrm{CDCl}_{3}, 300 \mathrm{MHz}\right) \delta: 8.07$ $(\mathrm{d}, J=8.4 \mathrm{~Hz}, 1 \mathrm{H}), 7.50(\mathrm{~m}, 2 \mathrm{H}), 6.83(\mathrm{~s}, 1 \mathrm{H}), 3.00(\mathrm{~m}$, 1H), 2.57 (m, 4H), $2.23(\mathrm{~m}, 3 \mathrm{H}), 1.96(\mathrm{~m}, 6 \mathrm{H}), 1.67$ (m, 9 $\mathrm{H})$; Anal. Calcd for $\mathrm{C}_{22} \mathrm{H}_{26} \mathrm{~F}_{3} \mathrm{NO}_{4} \mathrm{~S}$ : C 57.76, $\mathrm{H} 5.73, \mathrm{~N}$ 3.06. Found: C 57.99, H 5.72, N 3.10.

\section{(-)-3-Trifluoromethanesulfonyloxy-10-keto-N-}

methylmorphinan (1b) was obtained as yellow solid in $70.0 \%$ yield. M.p.: $123-124{ }^{\circ} \mathrm{C} ;{ }^{1} \mathrm{H} \mathrm{NMR}\left(\mathrm{CDCl}_{3}, 300\right.$ $\mathrm{MHz}) \delta: 8.17(\mathrm{~d}, J=7.8 \mathrm{~Hz}, 1 \mathrm{H}), 7.26(\mathrm{~d}, J=7.5 \mathrm{~Hz}, 1 \mathrm{H})$, $7.23(\mathrm{~s}, 1 \mathrm{H}), 3.03(\mathrm{~m}, 1 \mathrm{H}), 2.63(\mathrm{~m}, 1 \mathrm{H}), 2.38(\mathrm{~m}, 1 \mathrm{H})$, $2.34(\mathrm{~s}, 3 \mathrm{H}), 2.12(\mathrm{~m}, 1 \mathrm{H}), 1.98(\mathrm{~m}, 2 \mathrm{H}), 1.52(\mathrm{~m}, 6 \mathrm{H})$, 1.07 (m, 2H); ${ }^{13} \mathrm{C} \mathrm{NMR}\left(\mathrm{CDCl}_{3}, 75 \mathrm{MHz}\right) \delta: 194.2,153.8$, $148.5,134.8,128.5,119.5,119.4$, 68.2, 47.2, 46.8, 43.1, $40.9,38.5,36.3,26.0,25.7,21.6$.

\section{(-)-3-Trifluoromethanesulfonyloxy-10-keto-N-} cyclopropylmethylmorphinan (1c) was obtained as yellow solid in $72.8 \%$ yield. M.p.: $86-88{ }^{\circ} \mathrm{C}$; ${ }^{1} \mathrm{H} \mathrm{NMR}$ $\left(\mathrm{CDCl}_{3}, 300 \mathrm{MHz}\right) \delta: 8.10(\mathrm{~d}, J=9.0 \mathrm{~Hz}, 1 \mathrm{H}), 7.20(\mathrm{~m}$, 
2H), $3.28(\mathrm{~s}, 1 \mathrm{H}), 2.93(\mathrm{~d}, J=7.8 \mathrm{~Hz}, 1 \mathrm{H}), 2.62(\mathrm{dd}, J=$ 5.4, $12.3 \mathrm{~Hz}, 1 \mathrm{H}), 2.36$ (d, $J=13.5 \mathrm{~Hz}, 1 \mathrm{H}), 2.17$ (d, $J=$ $12.6 \mathrm{~Hz}, 1 \mathrm{H}), 1.96(\mathrm{~m}, 3 \mathrm{H}), 1.59(\mathrm{~m}, 6 \mathrm{H}), 1.13(\mathrm{~m}, 2 \mathrm{H})$, $0.87(\mathrm{~m}, 1 \mathrm{H}), 0.48$ (d, J = 7.2 Hz, 2H), $0.25(\mathrm{~m}, 1 \mathrm{H}), 0.06$ $(\mathrm{m}, 1 \mathrm{H}) ;{ }^{13} \mathrm{C} \mathrm{NMR}\left(\mathrm{CDCl}_{3}, 75 \mathrm{MHz}\right) \delta: 195.1,153.8$, 148.7 , 134.8, 128.4, 119.4, 119.3, 66.5, 60.2, 57.2, 46.8, $45.4,40.9,39.0,36.3,26.0,25.8,21.6,9.0,4.3,3.3$.

\section{(-)-3-Trifluoromethanesulfonyloxy-10-keto-N-(2-} phenylethyl)morphinan (1d) was obtained as yellow oil in $67.3 \%$ yield. ${ }^{1} \mathrm{H} \mathrm{NMR}\left(\mathrm{CDCl}_{3}, 300 \mathrm{MHz}\right) \delta: 8.18(\mathrm{~d}, J=$ $7.8 \mathrm{~Hz}, 1 \mathrm{H}), 7.18(\mathrm{~m}, 7 \mathrm{H}), 3.25(\mathrm{~m}, 1 \mathrm{H}), 2.94(\mathrm{~m}, 1 \mathrm{H})$, $2.77(\mathrm{~m}, 2 \mathrm{H}), 2.53(\mathrm{~m}, 1 \mathrm{H}), 2.34(\mathrm{~m}, 1 \mathrm{H}), 2.01(\mathrm{~m}, 3 \mathrm{H})$, $1.50(\mathrm{~m}, 6 \mathrm{H}), 1.18(\mathrm{~m}, 3 \mathrm{H}) ;{ }^{13} \mathrm{C} \mathrm{NMR}\left(\mathrm{CDCl}_{3}, 75 \mathrm{MHz}\right) \delta$ : $194.8,153.8,148.6,140.1,134.8,128.7,128.5,128.2$, $125.9,119.5,119.4,65.8,56.9,46.7,45.8,40.9,39.0,36.3$, $33.8,26.0,25.8,21.6$.

\section{(-)-3-Trifluoromethanesulfonyloxy-10-keto-N-(2-} naphthylmethyl)morphinan (1e) was obtained as oil in $86.7 \%$ yield. ${ }^{1} \mathrm{H}$ NMR $\left(\mathrm{CDCl}_{3}, 300 \mathrm{MHz}\right) \delta: 8.22$ (dd, $J=$ 2.4, 8.4 Hz, 1H), $7.80(\mathrm{~m}, 3 \mathrm{H}), 7.53(\mathrm{dd}, J=1.8,8.7 \mathrm{~Hz}$, 1H), $7.44(\mathrm{~m}, 2 \mathrm{H}), 7.27(\mathrm{~m}, 1 \mathrm{H}), 7.24(\mathrm{~m}, 2 \mathrm{H}), 3.92$ (d, J = $13.5 \mathrm{~Hz}, 1 \mathrm{H}), 3.51(\mathrm{~d}, J=13.5 \mathrm{~Hz}, 1 \mathrm{H}), 3.16(\mathrm{~d}, J=3.0$ $\mathrm{Hz}, 1 \mathrm{H}), 2.69$ (m, 1H), 2.35 (d, J=14.1 Hz, 1H), 2.06 (m, $5 \mathrm{H}), 1.36(\mathrm{~m}, 8 \mathrm{H}) ;{ }^{13} \mathrm{C} \mathrm{NMR}\left(\mathrm{CDCl}_{3}, 75 \mathrm{MHz}\right) \delta: 194.8$, $153.8,148.7,135.8,134.8,133.2,132.7,128.5,127.8$, $127.7,127.5,127.1,125.8,125.5,119.5,119.4,65.9,59.4$, $46.8,45.3,41.0,39.0,36.2,26.0,25.7,21.6$.

\section{(-)-3-Trifluoromethanesulfonyloxy-10-keto-N-(3-}

methyl-2-butenyl)morphinan (1f) was obtained as yellow oil in $88.3 \%$ yield. ${ }^{1} \mathrm{H} \mathrm{NMR}\left(\mathrm{CDCl}_{3}, 300 \mathrm{MHz}\right) \delta: 8.08(\mathrm{~d}$, $J=8.1 \mathrm{~Hz}, 1 \mathrm{H}), 7.16(\mathrm{~m}, 2 \mathrm{H}), 5.14(\mathrm{~m}, 1 \mathrm{H}), 3.08(\mathrm{~m}, 2 \mathrm{H})$, $2.82(\mathrm{~m}, 1 \mathrm{H}), 2.69(\mathrm{~m}, 1 \mathrm{H}), 2.28(\mathrm{~d}, J=13.5 \mathrm{~Hz}, 1 \mathrm{H}), 2.06$ $(\mathrm{d}, J=12.6 \mathrm{~Hz}, 1 \mathrm{H}), 1.87(\mathrm{~m}, 2 \mathrm{H}), 1.64(\mathrm{~s}, 3 \mathrm{H}), 1.55(\mathrm{~s}$, $3 \mathrm{H}), 1.36(\mathrm{~m}, 4 \mathrm{H}), 1.10(\mathrm{~m}, 4 \mathrm{H}) ;{ }^{13} \mathrm{C} \mathrm{NMR}\left(\mathrm{CDCl}_{3}, 75\right.$ $\mathrm{MHz}) \delta: 195.1,153.8,148.6,135.9,134.8,129.4,128.4$, 119.4, 119.3, 66.3, 52.9, 46.8, 45.0, 40.8, 39.0, 36.2, 26.0, $25.9,25.7,21.5,18.0$.

\section{(-)-3-Trifluoromethanesulfonyloxy-10-keto-N-(2-} methoxyethyl)morphinan (1g) was obtained as a yellow solid in $96.8 \%$ yield. M.p.: $76-78{ }^{\circ} \mathrm{C} ;{ }^{1} \mathrm{H} \mathrm{NMR}\left(\mathrm{CDCl}_{3}, 300\right.$ $\mathrm{MHz}) \delta: 8.18(\mathrm{~d}, J=8.1 \mathrm{~Hz}, 1 \mathrm{H}), 7.23(\mathrm{~m}, 2 \mathrm{H}), 3.61(\mathrm{~m}$, 1H), 3.51 (m, 1H), 3.37 (s, 3H), 3.17 (s, 1H), 2.80 (m, 2H), $2.53(\mathrm{~m}, 1 \mathrm{H}), 2.36$ (d, $J=13.5 \mathrm{~Hz}, 1 \mathrm{H}), 2.19$ (m, 1H), 2.02 $(\mathrm{m}, 2 \mathrm{H}), 1.50(\mathrm{~m}, 6 \mathrm{H}), 1.30(\mathrm{~m}, 3 \mathrm{H}) ;{ }^{13} \mathrm{C} \mathrm{NMR}\left(\mathrm{CDCl}_{3}, 75\right.$ $\mathrm{MHz})$ \&: 195.0, 153.8, 148.6, 134.7, 128.4, 119.4, 119.3, $118.6(\mathrm{q}, J=318.7 \mathrm{~Hz}), 69.5,66.0,58.8,57.2,54.4,46.3$, $45.9,40.7,38.8,36.2,25.9,25.7,21.5$.

3-Trifluoromethanesulfonyloxy-morphine (3): To a slurry of Morphine $\cdot \mathrm{H}_{2} \mathrm{O}(10 \mathrm{~g}, 32.8 \mathrm{mmol})$ in $\mathrm{CH}_{2} \mathrm{Cl}_{2}(500$ $\mathrm{mL})$ at $0^{\circ} \mathrm{C}$ was added a solution of $\mathrm{Et}_{3} \mathrm{~N}(7 \mathrm{~mL})$. The mixture was stirred for $1 \mathrm{~h}$ at ambient temperature, and then N-phenyltrifluoromethanesulfonimide (14 g, $39.4 \mathrm{mmol}$ ) was added. After being stirred for $24 \mathrm{~h}$, the reaction mixture was extracted with $10 \%$ aqueous $\mathrm{KHCO}_{3}(100$ $\mathrm{mL})$. The organic layer was dried $\left(\mathrm{Na}_{2} \mathrm{SO}_{4}\right)$, filtered, and concentrated in vacuo. The solid was dissolved in ether $(100 \mathrm{~mL})$, and extracted with $1 \mathrm{~N} \mathrm{HCl}(4 \times 120 \mathrm{~mL})$. The $\mathrm{pH}$ of the acidic layer was adjusted to 9 by adding ammonium hydroxide. The aqueous layer was then extracted with $\mathrm{CH}_{2} \mathrm{Cl}_{2}$ and the extracts were washed with brine, dried with $\mathrm{Na}_{2} \mathrm{SO}_{4}$, filtered and evaporated to get solid. The solid residue was further washed by anhydrous ether to give the triflate $\mathbf{3}^{3}$ as a white solid, $9.4 \mathrm{~g}(68.6 \%)$. m.p.: $121-123^{\circ} \mathrm{C}$; ${ }^{1} \mathrm{H}$ NMR $\left(\mathrm{CDCl}_{3}, 300 \mathrm{MHz}\right) \delta: 6.89(\mathrm{~d}, J=8.4 \mathrm{~Hz} 1 \mathrm{H})$, $6.64(\mathrm{~d}, J=8.4 \mathrm{~Hz} 1 \mathrm{H}), 5.70(\mathrm{~d}, J=10.2 \mathrm{~Hz}, 1 \mathrm{H}), 5.29$ (d, $J=9.6 \mathrm{~Hz} 1 \mathrm{H}), 5.02(\mathrm{~d}, J=6.3 \mathrm{~Hz}, 1 \mathrm{H}), 4.20(\mathrm{~m}, 1 \mathrm{H})$, $3.38(\mathrm{~m}, 1 \mathrm{H}), 3.05(\mathrm{~m}, 1 \mathrm{H}), 2.90$ (brs, 1H), $2.61(\mathrm{~m}, 2 \mathrm{H})$, $2.36(\mathrm{~m}, 5 \mathrm{H}), 2.09$ (m, 1H), 1.90 (d, J=11.4 Hz, 1H).

\section{(-)-3-Trifluoromethanesulfonyloxy-N-cyclopropyl}

methylmorphinan (5) was prepared according to the same procedure as described for the preparation of triflate $\mathbf{1}$ in $76 \%$ yield. ${ }^{1} \mathrm{H}$ NMR $\left(\mathrm{CDCl}_{3}, 300 \mathrm{MHz}\right) \delta: 7.16(\mathrm{~s}, 1 \mathrm{H})$, $7.12(\mathrm{~m}, 1 \mathrm{H}), 6.99(\mathrm{dd}, J=2.1,8.7 \mathrm{~Hz}, 1 \mathrm{H}), 3.1(\mathrm{~m}, 1 \mathrm{H})$, $2.96(\mathrm{~d}, J=18.9 \mathrm{~Hz}, 1 \mathrm{H}), 2.66(\mathrm{~m}, 2 \mathrm{H}), 2.46(\mathrm{~m}, 1 \mathrm{H}), 2.31$ $(\mathrm{m}, 2 \mathrm{H}), 1.85(\mathrm{~m}, 3 \mathrm{H}), 1.66(\mathrm{~d}, J=11.7 \mathrm{~Hz}, 1 \mathrm{H}), 1.56(\mathrm{~d}, J$ $=12.3 \mathrm{~Hz}, 1 \mathrm{H}), 1.37(\mathrm{~m}, 6 \mathrm{H}), 0.85(\mathrm{~m}, 1 \mathrm{H}), 0.50(\mathrm{~m}, 2 \mathrm{H})$, $0.11(\mathrm{~m}, 2 \mathrm{H}) ;{ }^{13} \mathrm{C} \mathrm{NMR}\left(\mathrm{CDCl}_{3}, 75 \mathrm{MHz}\right) \delta: 144.7,140.0$, 134.9, 125.6, 122.8 (q, $J=318.7 \mathrm{~Hz}), 114.5,114.4,56.3$, 51.8, 41.7, 41.0, 38.1, 34.6, 32.9, 23.1, 22.8, 20.7, 18.2, 5.8 .

3-Methyl-4-acetylphenyl triflate (7) was obtained as pale yellow oil in $98 \%$ yield. ${ }^{1} \mathrm{H} \mathrm{NMR}\left(\mathrm{CDCl}_{3}, 300 \mathrm{MHz}\right) \delta$ : $7.9(\mathrm{~m}, 1 \mathrm{H}), 7.84(\mathrm{~m}, 1 \mathrm{H}), 7.33(\mathrm{dd}, J=4.5,8.1 \mathrm{~Hz}, 1 \mathrm{H})$, $2.60(\mathrm{~s}, 3 \mathrm{H}), 2.43(\mathrm{~s}, 3 \mathrm{H})$.

4-Propionylphenyl triflate (9) was obtained as colorless oil in $97.4 \%$ yield. ${ }^{1} \mathrm{H} \mathrm{NMR}\left(\mathrm{CDCl}_{3}, 300 \mathrm{MHz}\right) \delta: 8.07$ (d, $J=7.2 \mathrm{~Hz}, 2 \mathrm{H}), 7.37(\mathrm{~d}, J=7.2 \mathrm{~Hz}, 2 \mathrm{H}), 3.02$ (q, $J=7.2$

(3) (a) Hedberg, M. H.; Johansson, A. M.; Hacksell, U. J. Chem. Soc., Chem. Commun. 1992, 845-846. (b) Hedberg, M. H.; Johansson, A. M.; Nordvall, G.; Yliniemela, A.; Li, H.-B.; Martin, A. R.; Hjorth, S.; Unelius, L.; Sundell, S.; Hacksell, U. J. Med. Chem. 1995, 38, 647-658. 
$\mathrm{Hz}, 2 \mathrm{H}), 1.23(\mathrm{t}, J=7.2 \mathrm{~Hz}, 3 \mathrm{H}) ;{ }^{13} \mathrm{C}$ NMR $\left(\mathrm{CDCl}_{3}, 75\right.$ MHz) $\delta: 198.7,152.2,136.6,121.5,118.6(\mathrm{q}, J=318.7$ $\mathrm{Hz}), 31.8,7.8$.

General Procedure for Microwave-Promoted cyanation. In a $10-\mathrm{mL}$ glass tube were placed triflate $(0.2 \mathrm{mmol})$, $\mathrm{Zn}(\mathrm{CN})_{2}$ (47 mg, $\left.0.4 \mathrm{mmol}\right), \mathrm{Pd}\left(\mathrm{Ph}_{3} \mathrm{P}\right)_{4}(19 \mathrm{mg}, 0.016$ $\mathrm{mmol}), 5 \mathrm{~mL}$ of DMF, and a magnetic stir bar. The vessel was sealed with a septum and placed into the microwave cavity. Microwave irradiation was used and the temperature was rapidly increased from room temperature to $200^{\circ} \mathrm{C}$. Once $200^{\circ} \mathrm{C}$ was reached, the reaction mixture was held at this temperature for $15 \mathrm{~min}$ and then cooled rapidly to room temperature. The reaction vessel was opened and the contents were poured into a separating funnel. Saturated sodium carbonate and water were added, and the mixture was extracted with ethyl acetate $(50 \mathrm{~mL} \times 3)$. The extracts were combined, washed with brine and dried over anhydrous sodium sulfate. After evaporation of the solvent, the residue was subjected to silica gel column chromatography to yield the corresponding nitrile compounds.

\section{(-)-3-Cyano-3-desoxy-10-keto-N-cyclobutylmethyl}

morphinan (2a) was obtained as a yellow viscous solid. ${ }^{1} \mathrm{H}$ NMR $\left(\mathrm{CDCl}_{3}, 300 \mathrm{MHz}\right) \delta: 8.11(\mathrm{~d}, J=7.8 \mathrm{~Hz}, 1 \mathrm{H})$, $7.64(\mathrm{~s}, 1 \mathrm{H}), 7.62(\mathrm{~d}, J=7.8 \mathrm{~Hz}, 1 \mathrm{H}), 3.08(\mathrm{~m}, 1 \mathrm{H}), 2.61$ (m, 3H), $2.41(\mathrm{~d}, J=13.8 \mathrm{~Hz}, 1 \mathrm{H}), 2.28(\mathrm{~m}, 1 \mathrm{H}), 1.96(\mathrm{~m}$, 6H), $1.69(\mathrm{~m}, 4 \mathrm{H}), 1.48(\mathrm{~m}, 5 \mathrm{H}), 1.13(\mathrm{~m}, 1 \mathrm{H}), 1.00(\mathrm{~m}$, $1 \mathrm{H}) ;{ }^{13} \mathrm{C} \mathrm{NMR}\left(\mathrm{CDCl}_{3}, 75 \mathrm{MHz}\right) \delta: 195.6,146.3,137.7$, 130.7, 129.6, 126.1, 118.2, 117.5, 66.7, 61.3, 46.4, 45.3, 40.9, 38.5, 36.0, 33.7, 27.3, 26.7, 26.0, 25.7, 21.7, 18.6; Anal. Calcd for $\mathrm{C}_{22} \mathrm{H}_{26} \mathrm{~N}_{2} \mathrm{O}$ : C 79.00, H 7.84, N 8.38. Found: C 78.83, H 8.12, N 8.36.

(-)-3-Cyano-3-desoxy-10-keto-N-methylmorphinan (2b) was obtained as a yellow solid. M.p.: $188-190{ }^{\circ} \mathrm{C} ;{ }^{1} \mathrm{H}$ NMR $\left(\mathrm{CDCl}_{3}, 300 \mathrm{MHz}\right) \delta: 8.13(\mathrm{~d}, J=8.4 \mathrm{~Hz}, 1 \mathrm{H}), 7.67(\mathrm{~s}$, 1H), $7.63(\mathrm{~d}, J=8.4 \mathrm{~Hz}, 1 \mathrm{H}), 3.05(\mathrm{~d}, J=2.7 \mathrm{~Hz}, 1 \mathrm{H})$, $2.65(\mathrm{~m}, 1 \mathrm{H}), 2.43(\mathrm{~d}, J=14.1 \mathrm{~Hz}, 1 \mathrm{H}), 2.33(\mathrm{~s}, 3 \mathrm{H}), 2.12$ (dt, $J=3.0,13.2 \mathrm{~Hz}, 1 \mathrm{H}), 1.97(\mathrm{~m}, 2 \mathrm{H}), 1.52(\mathrm{~m}, 5 \mathrm{H}), 1.16$ $(\mathrm{m}, 3 \mathrm{H}) ;{ }^{13} \mathrm{C} \mathrm{NMR}\left(\mathrm{CDCl}_{3}, 75 \mathrm{MHz}\right) \delta: 194.8,146.2$, 137.7, 130.8, 129.8, 126.3, 118.2, 117.7, 68.1, 47.1, 46.6, 43.1, 40.9, 38.1, 36.0, 26.0, 25.6, 21.7; Anal. Calcd for $\mathrm{C}_{18} \mathrm{H}_{20} \mathrm{~N}_{2} \mathrm{O} .0 .2 \mathrm{H}_{2} \mathrm{O}: \mathrm{C} 76.13, \mathrm{H} 7.24, \mathrm{~N}$ 9.87. Found: C 76.45, H 7.16, N 9.86.

\section{(-)-3-Cyano-3-desoxy-10-keto-N-cyclopropylmethyl} morphinan (2c) was obtained as a yellow solid. M.p.: 114-
$116{ }^{\circ} \mathrm{C} ;{ }^{1} \mathrm{H}$ NMR $\left(\mathrm{CDCl}_{3}, 300 \mathrm{MHz}\right) \delta: 8.07(\mathrm{~d}, J=7.8 \mathrm{~Hz}$, $1 \mathrm{H}), 7.64(\mathrm{~s}, 1 \mathrm{H}), 7.58(\mathrm{~d}, J=7.8 \mathrm{~Hz}, 1 \mathrm{H}), 3.28(\mathrm{~d}, J=3.0$ $\mathrm{Hz}, 1 \mathrm{H}), 2.90(\mathrm{~m}, 1 \mathrm{H}), 2.58(\mathrm{dd}, J=5.7,12.9 \mathrm{~Hz}, 1 \mathrm{H})$, $2.40(\mathrm{~d}, J=13.8 \mathrm{~Hz}, 1 \mathrm{H}), 2.15(\mathrm{dt}, J=3.3,13.2 \mathrm{~Hz}, 1 \mathrm{H})$, $1.94(\mathrm{~m}, 3 \mathrm{H}), 1.50(\mathrm{~m}, 6 \mathrm{H}), 1.10(\mathrm{~m}, 3 \mathrm{H}), 0.48(\mathrm{~m}, 2 \mathrm{H})$, $0.22(\mathrm{~m}, 2 \mathrm{H}), 0.05(\mathrm{~m}, 1 \mathrm{H}) ;{ }^{13} \mathrm{C} \mathrm{NMR}\left(\mathrm{CDCl}_{3}, 75 \mathrm{MHz}\right) \delta$ : 195.7, 146.4, 137.7, 130.7, 129.7, 126.2, 118.3, 117.6, $66.4,60.1,46.5,45.3,40.9,38.7,36.0,26.0,25.7,21.7$, 8.9, 4.2, 3.3; Anal. Calcd for $\mathrm{C}_{21} \mathrm{H}_{24} \mathrm{~N}_{2} \mathrm{O}$ : C 78.71, H 7.55, N 8.74. Found: C 78.62, H 7.57, N 8.61.

\section{(-)-3-Cyano-3-desoxy-10-keto-N-(2-phenylethyl)}

morphinan (2d) was obtained as yellow solid. ${ }^{1} \mathrm{H}$ NMR $\left(\mathrm{CDCl}_{3}, 300 \mathrm{MHz}\right) \delta: 8.11(\mathrm{~d}, J=7.8 \mathrm{~Hz}, 1 \mathrm{H}), 7.66(\mathrm{~d}, J=$ $1.2 \mathrm{~Hz}, 1 \mathrm{H}), 7.60(\mathrm{dd}, J=1.5,7.8 \mathrm{~Hz}, 1 \mathrm{H}), 7.23(\mathrm{~m}, 5 \mathrm{H})$, $3.26(\mathrm{~m}, 1 \mathrm{H}), 2.92(\mathrm{~m}, 1 \mathrm{H}), 2.74(\mathrm{~m}, 2 \mathrm{H}), 2.50(\mathrm{~m}, 1 \mathrm{H})$, $2.44(\mathrm{~d}, J=6.9 \mathrm{~Hz}, 1 \mathrm{H}), 2.12(\mathrm{~m}, 1 \mathrm{H}), 1.97(\mathrm{~m}, 1 \mathrm{H}), 1.49$ $(\mathrm{m}, 6 \mathrm{H}), 1.10(\mathrm{~m}, 3 \mathrm{H}) ;{ }^{13} \mathrm{C} \mathrm{NMR}\left(\mathrm{CDCl}_{3}, 75 \mathrm{MHz}\right) \delta$ : $195.5,146.3,140.0,137.7,130.8,129.8,128.7,128.2$, $126.3,126.0,118.3,117.7,65.8,56.9,46.4,45.7,40.9$, 38.6, 36.0, 33.8, 26.0, 25.8, 21.7; Anal. Calcd for $\mathrm{C}_{25} \mathrm{H}_{26} \mathrm{~N}_{2} \mathrm{O} .1 / 4 \mathrm{H}_{2} \mathrm{O}: \mathrm{C} 80.07, \mathrm{H}$ 7.12, N 7.47. Found: $\mathrm{C}$ $80.32, \mathrm{H} 8.15$, N 7.41 .

\section{(-)-3-Cyano-3-desoxy-10-keto-N-(2-naphthylmethyl)} morphinan (2e) was obtained as yellow solid. ${ }^{1} \mathrm{H}$ NMR $\left(\mathrm{CDCl}_{3}, 300 \mathrm{MHz}\right) \delta: 8.18(\mathrm{~d}, J=7.8 \mathrm{~Hz}, 1 \mathrm{H}), 7.79(\mathrm{~m}$, $4 \mathrm{H}), 7.65(\mathrm{~m}, 2 \mathrm{H}), 7.53(\mathrm{~d}, J=8.1 \mathrm{~Hz}, 1 \mathrm{H}), 7.45(\mathrm{~m}, 2 \mathrm{H})$, $3.90(\mathrm{~d}, J=13.5 \mathrm{~Hz}, 1 \mathrm{H}), 3.52(\mathrm{~d}, J=13.5 \mathrm{~Hz}, 1 \mathrm{H}), 3.18$ (s, 1H), $2.68(\mathrm{~d}, J=8.7 \mathrm{~Hz}, 1 \mathrm{H}), 2.42(\mathrm{~d}, J=14.1 \mathrm{~Hz}, 1 \mathrm{H})$, $2.18(\mathrm{~d}, J=12.9 \mathrm{~Hz}, 1 \mathrm{H}), 1.99(\mathrm{~m}, 2 \mathrm{H}), 1.45(\mathrm{~m}, 8 \mathrm{H}) ;{ }^{13} \mathrm{C}$ NMR $\left(\mathrm{CDCl}_{3}, 75 \mathrm{MHz}\right) \delta$ : 195.5, 146.4, 137.7, 135.7, $133.2,132.7,130.8,129.8,127.9,127.7,127.5,127.1$, $126.3,125.9,125.6,118.3,117.7,65.9,59.4,46.5,45.2$, 41.0, 38.6, 36.0, 26.0, 25.6, 21.7; Anal. Calcd for $\mathrm{C}_{28} \mathrm{H}_{26} \mathrm{~N}_{2} \mathrm{O}: \mathrm{C} 82.73, \mathrm{H} 6.45, \mathrm{~N}$ 6.89. Found: C 82.45, H $6.47, \mathrm{~N} 6.88$.

\section{(-)-3-Cyano-3-desoxy-10-keto-N-(3-methyl-2-butenyl)} morphinan (2f) was obtained as yellow oil. ${ }^{1} \mathrm{H}$ NMR $\left(\mathrm{CDCl}_{3}, 300 \mathrm{MHz}\right) \delta: 8.05(\mathrm{dd}, J=2.0,7.8 \mathrm{~Hz}, 1 \mathrm{H}), 7.58$ $(\mathrm{m}, 2 \mathrm{H}), 5.12(\mathrm{t}, J=6.9 \mathrm{~Hz}, 1 \mathrm{H}), 3.10(\mathrm{~m}, 2 \mathrm{H}), 2.80(\mathrm{~m}$, $1 \mathrm{H}), 2.68(\mathrm{~m}, 1 \mathrm{H}), 2.34(\mathrm{~d}, J=14.1 \mathrm{~Hz}, 1 \mathrm{H}), 2.05(\mathrm{~m}, 1 \mathrm{H})$, $1.86(\mathrm{~m}, 2 \mathrm{H}), 1.64(\mathrm{~s}, 3 \mathrm{H}), 1.57$ (s, 3H), $1.40(\mathrm{~m}, 5 \mathrm{H}), 1.16$ $(\mathrm{m}, 3 \mathrm{H}) ;{ }^{13} \mathrm{C} \mathrm{NMR}\left(\mathrm{CDCl}_{3}, 75 \mathrm{MHz}\right) \delta: 195.8,146.4$, $137.7,135.9,130.7,129.7,126.2,120.7,118.3,117.6$, 66.3, 52.9, 46.6, 44.9, 40.9, 38.7, 36.0, 26.0, 25.9, 25.7, 21.7, 18.0; Anal. Calcd for $\mathrm{C}_{22} \mathrm{H}_{26} \mathrm{~N}_{2} \mathrm{O} .0 .5 \mathrm{H}_{2} \mathrm{O}: \mathrm{C} 76.93, \mathrm{H}$ 7.92, N 8.16. Found: C 76.54, H 7.82, N 7.88. 
(-)-3-Cyano-3-desoxy-10-keto-N-(2-methoxyethyl)

morphinan (2g) was obtained as a yellow viscous solid.

${ }^{1} \mathrm{H}$ NMR $\left(\mathrm{CDCl}_{3}, 300 \mathrm{MHz}\right) \delta: 8.11(\mathrm{~m}, 1 \mathrm{H}), 7.65(\mathrm{~m}, 2 \mathrm{H})$, $3.53(\mathrm{~m}, 2 \mathrm{H}), 3.36(\mathrm{~s}, 3 \mathrm{H}), 3.18(\mathrm{~s}, 1 \mathrm{H}), 2.78(\mathrm{~m}, 2 \mathrm{H}), 2.47$

(m, 2H), $2.19(\mathrm{~m}, 1 \mathrm{H}), 2.01(\mathrm{~m}, 2 \mathrm{H}), 1.57(\mathrm{~m}, 5 \mathrm{H}), 1.08$

$(\mathrm{m}, 3 \mathrm{H}) ;{ }^{13} \mathrm{C} \mathrm{NMR}\left(\mathrm{CDCl}_{3}, 75 \mathrm{MHz}\right) \delta: 195.7,146.4$, 137.6, 130.8, 129.8, $126.2118 .3,117.7,69.6,66.0,58.9$, 54.5, 46.2, 45.8, 40.7, 38.5, 36.0, 26.0, 25.7, 21.7; Anal. Calcd for $\mathrm{C}_{20} \mathrm{H}_{24} \mathrm{~N}_{2} \mathrm{O}_{2}$ : C 74.04, H 7.46, N 8.64. Found: C 74.00, H 7.53, N 8.41.

3-Cyano-3-desoxy-morphine (4) was obtained as a pale yellow solid. M.p.: $191-192{ }^{\circ} \mathrm{C} ;{ }^{1} \mathrm{H}$ NMR $\left(\mathrm{CDCl}_{3}, 300\right.$ MHz) $\delta: 7.20(\mathrm{~d}, J=8.1 \mathrm{~Hz} 1 \mathrm{H}), 6.68(\mathrm{~d}, J=8.1 \mathrm{~Hz} 1 \mathrm{H})$, $5.71(\mathrm{~d}, J=9.0 \mathrm{~Hz}, 1 \mathrm{H}), 5.30(\mathrm{~d}, J=8.1 \mathrm{~Hz} 1 \mathrm{H}), 5.02(\mathrm{~d}, J$ $=5.7 \mathrm{~Hz}, 1 \mathrm{H}), 4.23(\mathrm{~s}, 1 \mathrm{H}), 3.37(\mathrm{~m}, 1 \mathrm{H}), 3.12(\mathrm{~m}, 1 \mathrm{H})$, $2.64(\mathrm{~m}, 1 \mathrm{H}), 2.35(\mathrm{~m}, 10 \mathrm{H}) ;{ }^{13} \mathrm{C} \mathrm{NMR}\left(\mathrm{CDCl}_{3}, 75 \mathrm{MHz}\right)$ $\delta: 144.7,141.3,133.4,131.3,130.7,128.4,123.5,119.8$, 115.8, 66.8, 58.1, 45.8, 43.0, 42.6, 41.3, 40.6, 35.3, 21.6; Anal. Calcd for $\mathrm{C}_{18} \mathrm{H}_{18} \mathrm{~N}_{2} \mathrm{O}_{2} .1 .0 \mathrm{H}_{2} \mathrm{O}: \mathrm{C} 69.21, \mathrm{H} 6.45, \mathrm{~N}$ 8.97. Found: $\mathrm{C} 69.22$, H 6.12, N 8.53.

(-)-3-Cyano-3-desoxy-N-cyclopropyl methylmorphinan (6) was obtained as pale yellow oil. ${ }^{1} \mathrm{H} \mathrm{NMR}\left(\mathrm{CDCl}_{3}, 300\right.$ MHz) $\delta: 7.53(\mathrm{~d}, J=1.5,1 \mathrm{H}), 7.38(\mathrm{dd}, J=1.8,8.1,1 \mathrm{H})$, $7.20(\mathrm{~d}, J=8.1 \mathrm{~Hz}, 1 \mathrm{H}), 3.0(\mathrm{~m}, 1 \mathrm{H}), 2.97(\mathrm{~m}, 1 \mathrm{H}), 2.66$ $(\mathrm{m}, 2 \mathrm{H}), 2.40(\mathrm{~m}, 1 \mathrm{H}), 2.29(\mathrm{~m}, 2 \mathrm{H}), 1.83(\mathrm{~m}, 3 \mathrm{H}), 1.60(\mathrm{~d}$, $J=12.0 \mathrm{~Hz}, 1 \mathrm{H}), 1.56(\mathrm{~m}, 1 \mathrm{H}), 1.33(\mathrm{~m}, 6 \mathrm{H}), 0.87(\mathrm{~m}$, $1 \mathrm{H}), 0.55(\mathrm{~m}, 2 \mathrm{H}), 0.10(\mathrm{~m}, 2 \mathrm{H}) ;{ }^{13} \mathrm{C} \mathrm{NMR}\left(\mathrm{CDCl}_{3}, 75\right.$ MHz) $\delta: 144.3,142.6,129.8,128.8,128.7,119.7,110.1$, 61.6, 55.7, 44.9, 42.0, 38.0, 36.4, 34.9, 27.8, 26.9, 26.6, 25.6, 19.0, 7.2; GC-MS: $306\left(\mathrm{M}^{+}+1\right)$; Anal. Calcd for $\mathrm{C}_{21} \mathrm{H}_{26} \mathrm{~N}_{2} \cdot 0.5 \mathrm{H}_{2} \mathrm{O}: \mathrm{C} 79.96, \mathrm{H}$ 8.63, N 8.88. Found: C 79.53, H 8.70, N 8.93.

3-Methyl-4-acetylbenzonitrile (8) was obtained as white solid. M.p.: $76-78{ }^{0} \mathrm{C} ;{ }^{1} \mathrm{H}$ NMR $\left(\mathrm{CDCl}_{3}, 300 \mathrm{MHz}\right) \delta: 7.89$ (s, 1H), $7.84(\mathrm{~d}, J=5.1 \mathrm{~Hz}, 1 \mathrm{H}), 7.72(\mathrm{~d}, J=5.1 \mathrm{~Hz}, 1 \mathrm{H})$, $2.63(\mathrm{~s}, 6 \mathrm{H}) ;{ }^{13} \mathrm{C} \mathrm{NMR}\left(\mathrm{CDCl}_{3}, 75 \mathrm{MHz}\right) \delta: 196.9,142.5$, 139.7, 132.8, 129.6, 125.8, 117.2, 116.7, 26.7, 20.5; Anal. Calcd for $\mathrm{C}_{10} \mathrm{H}_{9} \mathrm{NO}$ : C 75.45, H 5.70, N 8.80. Found: C 75.15, H 5.66, N 8.77.

4-Propionylbenzonitrile (10) ${ }^{4}$ was obtained as a white solid. ${ }^{1} \mathrm{H} \mathrm{NMR}\left(\mathrm{CDCl}_{3}, 300 \mathrm{MHz}\right) \delta: 8.06(\mathrm{~d}, J=7.8 \mathrm{~Hz}$,
2H), $7.78(\mathrm{~d}, J=7.8 \mathrm{~Hz}, 2 \mathrm{H}), 3.03(\mathrm{q}, J=6.9 \mathrm{~Hz}, 2 \mathrm{H})$, $1.24(\mathrm{t}, J=6.9 \mathrm{~Hz}, 3 \mathrm{H}) ;{ }^{13} \mathrm{C} \mathrm{NMR}\left(\mathrm{CDCl}_{3}, 75 \mathrm{MHz}\right) \delta$ : 199.2, 139.7, 132.4, 128.3, 117.9, 116.1, 32.1, 7.8.

(4) Russell, G. A.; Mudryk, B.; Jawdosiuk, M.; Wrobel, Z. pp 1879 - 1884 J. Org. Chem. 1982, 47, 1879-1884. 
